\title{
The techniques of uniportal video-assisted thoracoscopic surgery: lower lobectomies and lymphadenectomy
}

\author{
Carlos Galvez ${ }^{1}$, Julio Sesma ${ }^{1}$, Sergio Bolufer ${ }^{1}$, Francisco Lirio ${ }^{2}, J_{o n e}$ del Campo ${ }^{1}$, Sergio Maroto ${ }^{1}$, \\ Juan Manuel Corcoles ${ }^{3}$ \\ ${ }^{1}$ Department of Thoracic Surgery, University General Hospital, Alicante, Spain; ${ }^{2}$ Department of Thoracic Surgery, Hospital of Denia, Denia, \\ Alicante, Spain; ${ }^{3}$ Department of Thoracic Surgery, University Hospital of Vinalopo, Alicante, Spain \\ Correspondence to: Carlos Galvez, MD, PhD. C/Pintor Baeza, 12. 03010, Alicante, Spain. Email: carlos.galvez.cto@gmail.com.
}

\begin{abstract}
Uniportal video-assisted thoracoscopic surgery (VATS) is probably the most successful singleincision approach worldwide, probably secondary to several specific circumstances: multiportal VATS was hardly getting his recognition in the thoracic surgeon's community; the extraordinary effort by his creators and believers for developing the technique and giving massive diffusion; the subjective feeling by surgeons who performed the approach about its benefits and advantages. Despite this, many efforts have focused on extending new indications and describing variations of the original intercostal uniportal VATS, but few quality papers have analyzed the real impact of the approach and its real advantages or disadvantages comparing to multiportal VATS. Thus, many surgeons still feel little confidence on the approach and reject his performance. With the aim of standardizing the approach and the technical aspects for nonexperienced or beginners, the Uniportal VATS Interest Group (UVIG) of the European Society of Thoracic Surgery (ESTS) decided to set the basis for homogenization of the technique to cement the development of high-level evidence works that shed light on the real outcomes of uniportal compared to multiportal VATS. This article describes the main specific technical aspects while performing lower lobectomies and lymphadenectomy, which were described as the most suitable cases for initiating the learning curve.
\end{abstract}

Keywords: Thoracic surgery; video-assisted; lung cancer surgery

Submitted Nov 15, 2018. Accepted for publication Jan 24, 2019.

doi: $10.21037 /$ jtd.2019.02.67

View this article at: http://dx.doi.org/10.21037/jtd.2019.02.67

\section{Introduction}

Pulmonary anatomical resections through a single-incision started in 2010 (1), and since then have experienced a huge worldwide spread secondary not only to natural diffusion of the technique, but also for the emphasis and support of developers and "believers" (2-9). Despite its amazing diffusion within these 8 years, there is still a lack of standardization of the technique, its actual indications and recommendations. In a mandatory effort from the Uniportal VATS Interest Group (UVIG) of the European Society of Thoracic Surgeons (ESTS), some experts in the technique are developing guidelines that should delimit the framework of application of uniportal video-assisted thoracoscopic surgery (VATS).
Lower lobectomies were described as more suitable for beginners in uniportal VATS because they show less anatomical variations than upper lobectomies. The objective of this article is to set the features, but also the tips and tricks for performing anatomical lower lobectomies, and how to perform the lymphadenectomy through this approach. Authors will describe essential aspects and also will recommend some tricks for less experienced surgeons in the approach.

\section{Starting point-VATS experience}

Initially some uniportal surgeons recommend that before starting a learning curve in uniportal VATS, surgeons should be experienced in multiportal VATS, but this is not 


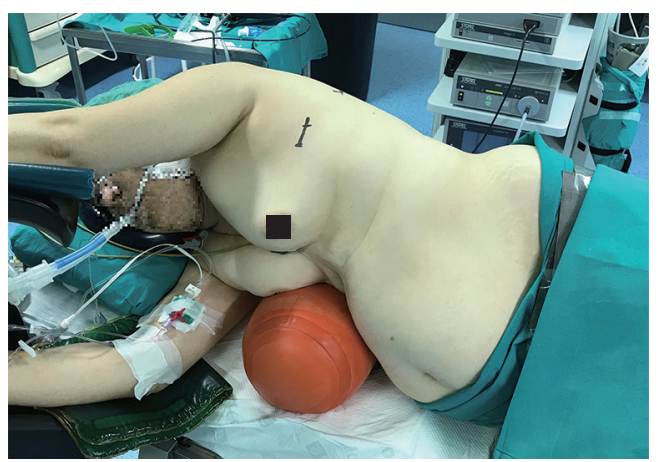

Figure 1 Patient positioning during left-lower lobectomy, with an inflatable roller behind the contralateral side.

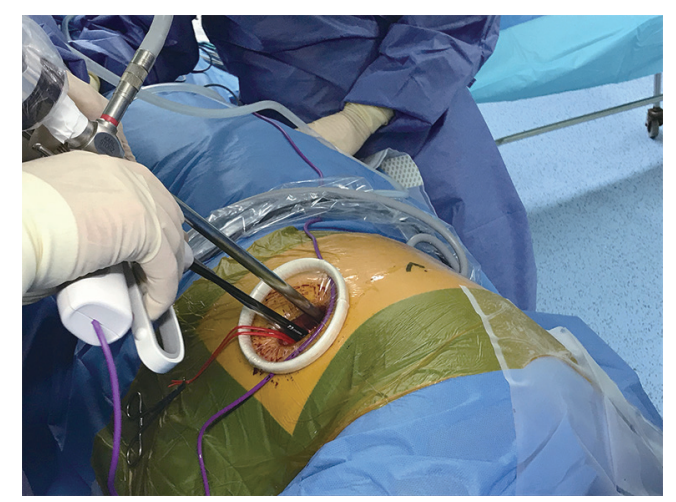

Figure 2 Instruments position: throughout most of the procedure, the camera stands in the upper part of the incision, and uniportal instruments and energy devices are placed in the lower part.

completely true. Some surgeons have shown feasible his training in uniportal VATS from open approach $(10,11)$. There are some interesting differences in geometrics between multiportal and uniportal VATS that make these approaches quite different (12), thus although useful for instrument handling, previous multiportal VATS knowledge can even difficult the acquisition of new concepts in uniportal handling. There is already some limited evidence that surgeons without previous multiportal VATS experience select more strictly his first cases and perform more cases by open approach during their learning curve, and this curve takes longer than previously experienced surgeons in VATS (13).

Moreover, uniportal VATS appeared in the age of technological development and "millennials", so we should keep in mind that new generations of surgeons have grown in video-assisted manipulation since they were kids and have developed skills that seem quite difficult for older surgeons.

Mentorship by an experienced uniportal surgeon seems advisable while performing first cases through this approach, in order to guide the acquisition of skills and manage the situation should a complication occurs.

\section{Patient setting and instrumental}

Patient should be placed in lateral decubitus, in a comfortable position for avoiding brachial plexus or shoulder injury, and we find useful two methods for slightly widening the intercostal space: operating tables than can be flexed, and we use inflatable pneumatic roller/ semirigid roller behind the contralateral side (Figure 1). This contralateral mild flexion makes more comfortable instrumental handling during the procedure.

A 4-cm incision for uniportal VATS lower lobectomies can be placed in the $5^{\text {th }}$ or $6^{\text {th }}$ intercostal space. Our recommendation for slim patients with large chest is the $6^{\text {th }}$ intercostal space, which results more comfortable because you enter the cavity just in front of the fissure and not from above, making easier the dissection. For obese patients we prefer the $5^{\text {th }}$ intercostal space to avoid a very low incision, because the diaphragm lies much more higher in these patients.

There are some anatomical variations in the exact location of the incision: some surgeons prefer more anterior incisions and other surgeons choose a more posterior incision. In our experience we find more ergonomic for surgeon's handling a slightly anterior incision than a posterior one, to avoid excessive elevation of both arms with its potential shoulder pain after long lasting procedures.

Wound protectors are really useful to prevent the thoracoscope of getting dirty from subcutaneous fatty tissue, specially in obese patients, and do not damage the intercostal nerve.

We recommend positioning the camera in the upper part of the incision for most of the procedure with a "flying-like" view, so as the surgeon can insert uniportal instruments and energy devices behind (Figure 2). In some specific steps, as dividing fissures, we sometimes switch and position the camera in the lower part, but just temporary.

There is one main difference in surgeon's position during lower lobectomies: while in upper lobectomies the assistant can stand in a more caudal position than the surgeon throughout the whole procedure (Figure 3A), for lower lobectomies we find quite useful the alternation between surgeon and assistant from right-to-left. For pulmonary 
A

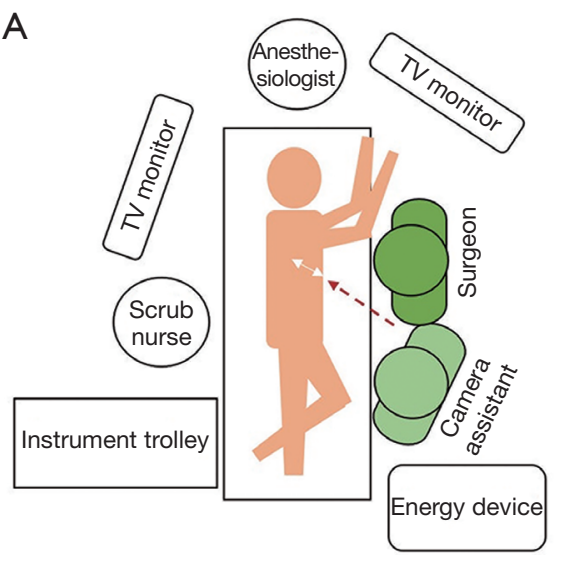

B

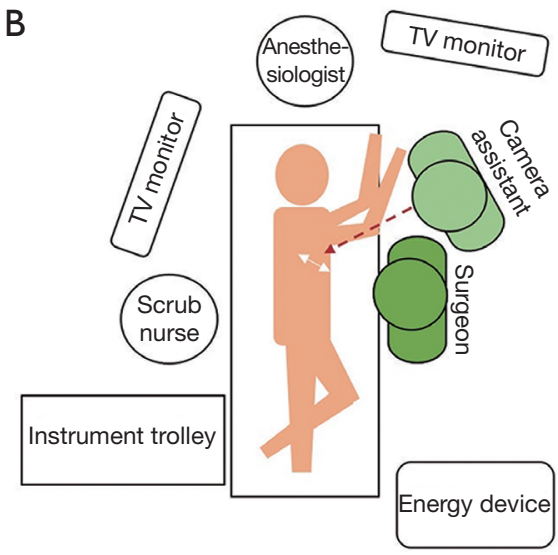

Figure 3 Surgeon, camera assistant and scrub nurse positioning. (A) The assistant stands caudally to the surgeon during most steps of the procedure; (B) for specific steps in the lower part of the chest, the assistant places cranially to the surgeon to avoid continuous crashing with his instruments.

ligament, inferior pulmonary vein dissection/division and subcarinal lymphadenectomy we prefer that the assistant places in a more cranial position, very close to patient's arms so as the surgeon can direct the lower part of the chest without continuous crashing with the thoracoscope (Figure 3B). Other surgeons prefer that the assistant stands in the opposite side of the patient but it needs longer training to develop skills for camera assistance in a specular image. The use of 30 degrees thoracoscopes makes easier avoiding continuous crashing between the surgeon's instrumental and the camera. The scrub nurse always stands in the opposite side of the patient close to his legs, so she/he can watch the secondary monitor behind the surgeon, and assist easily both the surgeon and the assistant.

There is a wide range of specific uniportal VATS/ VATS instrumental that has been specifically designed for this purpose throughout last 8 years. Main features of this specific instruments are its length (longer than open instrumental but shorter than laparoscopic one), double articulation (one inside the chest and other outside the incision), and its tailored design for uniportal purpose, usually angulating the tip to make easier the view from a frontal view like uniportal VATS. Although we initially used several instruments for dissection and division of tissue layers (combinations of dissectors, Metzenbaum scissors), the advent of energy devices such as bipolar Ligasure Maryland or ultrasonic Harmonic Scalpel have brought new and simpler options for both dissecting and dividing the bronchovascular structures and fissures.

\section{Right lower lobectomy}

We set a difference between those lower lobectomies with complete or almost complete fissure, and those with incomplete fissure.

\section{Complete or almost complete fissure [I-II Craig Walker classification (14)] (Figure 4)}

Lower lobectomies with complete fissure are easier procedures than upper lobectomies, because arterial division can be frequently performed in one unique step. After careful initial exploration, we first attempt the arterial dissection and division in the fissure. We prefer not to grasp the remaining lobes [right upper lobe (RUL) and right middle lobe (RML)], so the surgeon grasps lower lobe downwards while the assistant pulls bluntly with a sponge-stick the RUL/RML while dissecting the artery in the fissure. We first identify the artery, and then try to discover both the upper segment artery (A6) and the trunk for basilar segments. Then we divide both the anterior and the posterior fissure with energy devices or preferably endostaplers. Dividing anterior/posterior fissures makes easier and safer arterial dissection and division, but it's not mandatory and can't be done later. Division of posterior fissure can be safely done if surgeon divides first the pulmonary ligament and the posterior mediastinal pleura, so then he can tunnel from the fissure (just immediately behind the upper segment artery) to the posterior mediastinal pleura, always keeping in mind 


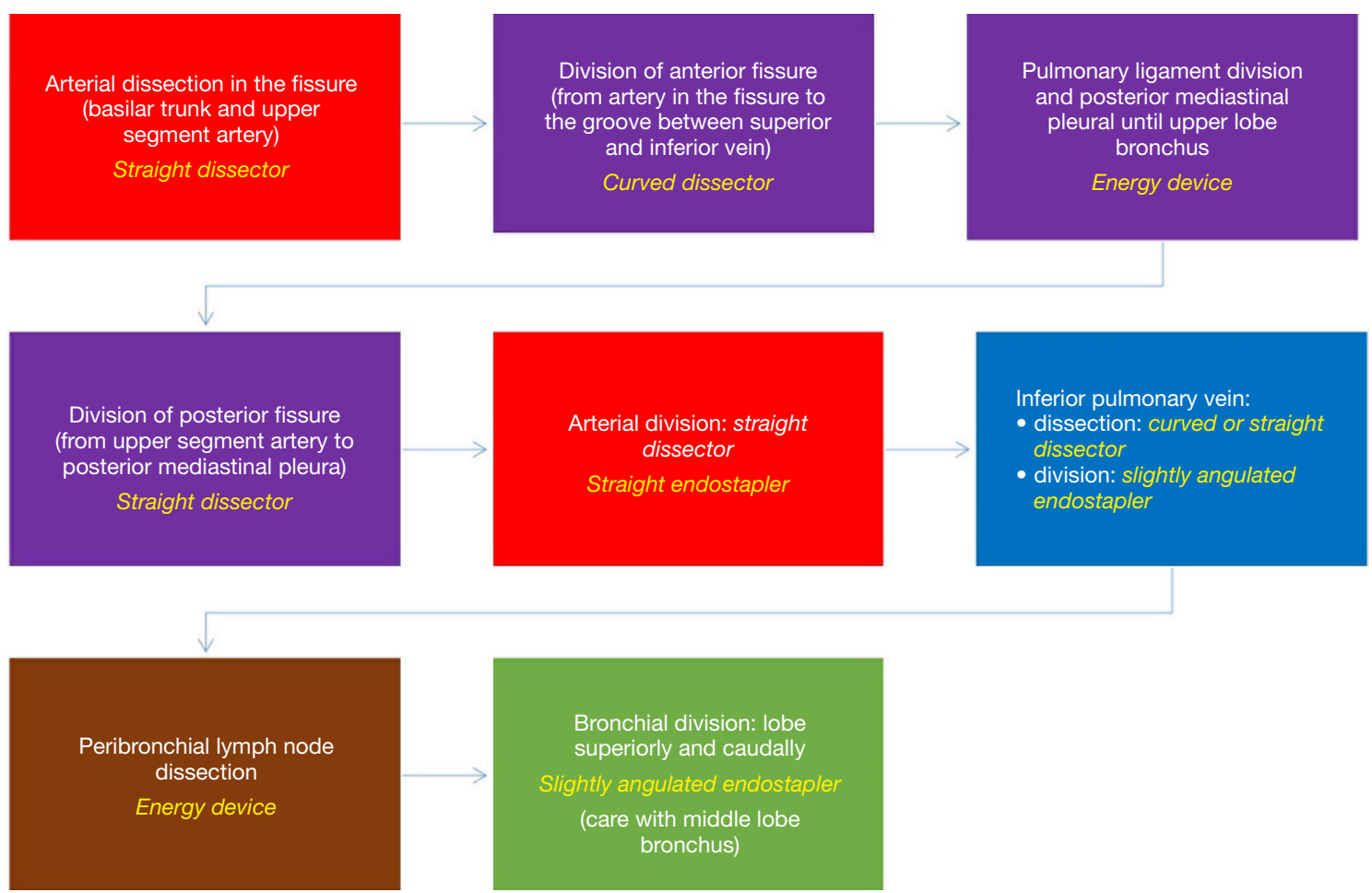

Figure 4 Right-lower lobectomy (complete fissure).

below the intermediate bronchus. Fissure division makes easier the dissection of the pulmonary artery from the bronchus, where the use of straight dissectors is better than usual curved dissectors. Then we complete the arterial division, if possible, with a unique vascular stapler including both A6 and basilar trunk (Figure 5A), but if necessary, it can be performed separately, even with the use of endoscopic clips for upper segment artery. Keep in mind that up to $17.4 \%$ of cases present with more than one arterial branch for the upper segment (S6) (15). In this step it's important to identify the middle lobe artery on the right in order to preserve it, and to remove the interlobar lymph nodes between middle-lobe and lowerlobe bronchus (LLB) (level 11).

For pulmonary ligament and inferior pulmonary vein division we prefer that the assistant with the camera places in a cranial position close to the flexed arms. We divide the ligament and then continue dissecting towards the subcarinal station until the posterior aspect of the upper-lobe bronchus. This way you expose the posterior aspect of the inferior vein and identify the vein for the upper segment (Figure 5B).

We always check that the middle lobe vein comes from the upper pulmonary vein, because anatomical variation coming from the lower vein is discovered in up to $7.1 \%$ of cases (15). Then, by pulling the lobe upwards towards the chest wall, surgeon can dissect anteriorly the vein easily. We find also useful a straight dissector for this step, but in some cases with big tumors where pulling upwards the lobe is difficult, a curved dissector can be preferable (Figure 5C). The surgeon can then divide the vein with a vascular stapler: place the stapler without angulation or just mild angulation of the tip upwards to avoid the posterior mediastinum with the tip of the stapler, and once positioned use the suction to pull the lobe anteriorly towards the stapler (Figure 5D).

After vascular divisions, bronchus must be attempted: pulling the lobe upwards towards the chest wall let the surgeon dissect peribronchial lymph nodes to expose the bronchus. Pulling the lobe towards the chest wall and caudal, surgeon can easily place the thick load stapler, (some angulation may be required), and it's important to check that the stapler is not compressing the carina between middle-lobe bronchus (MLB) and LLB by ventilating for a short while (Figure 6).

Sometimes surgeon can divide the artery/arteries without dividing the posterior fissure, thus when he gets to 

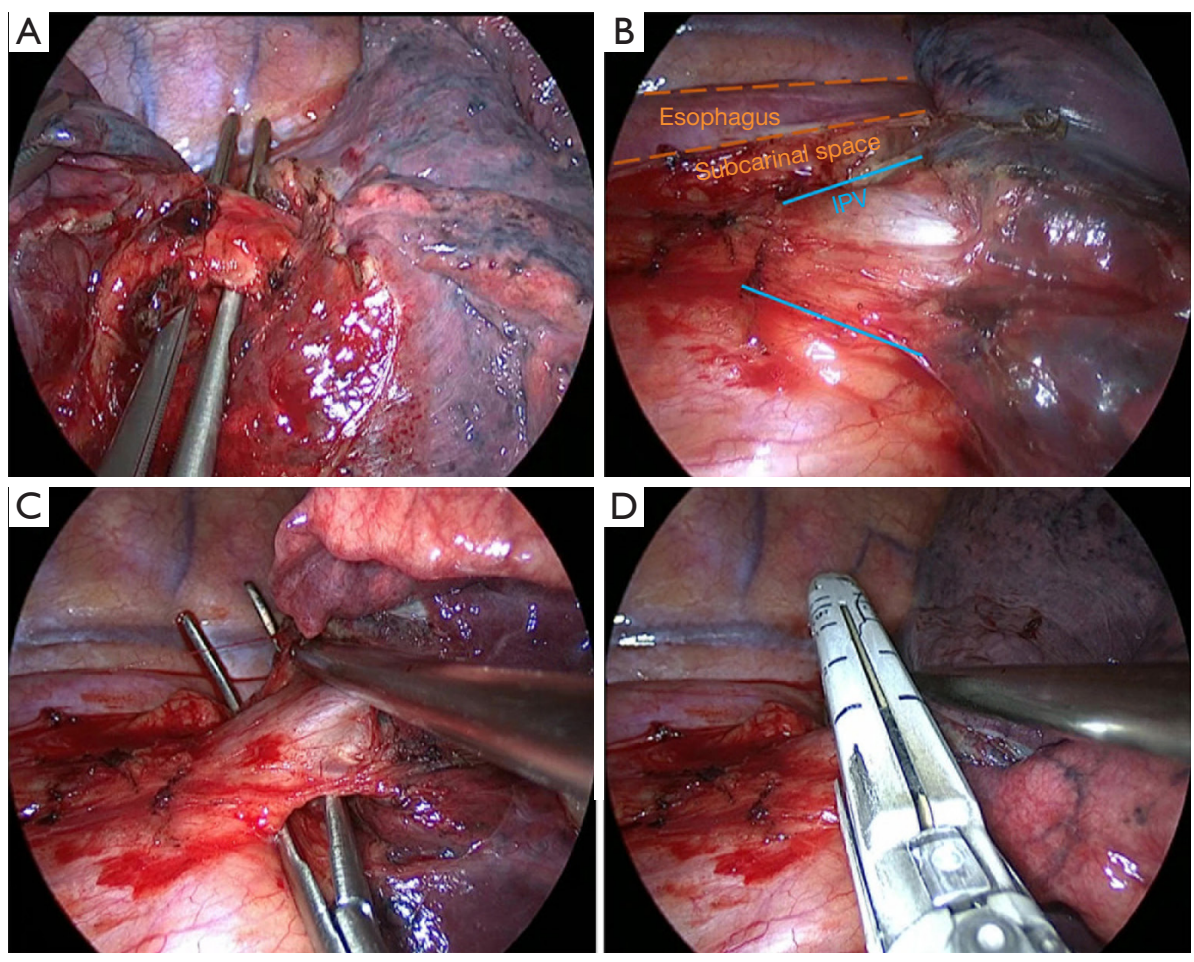

Figure 5 Right-lower lobectomy. (A) Combined dissection of both upper segment artery and basilar trunk; (B) posterior dissection of the inferior pulmonary vein (IPV) reaching the subcarinal space; (C) dissection of the IPV with a curved dissector; (D) suction assistance while dividing the IPV.

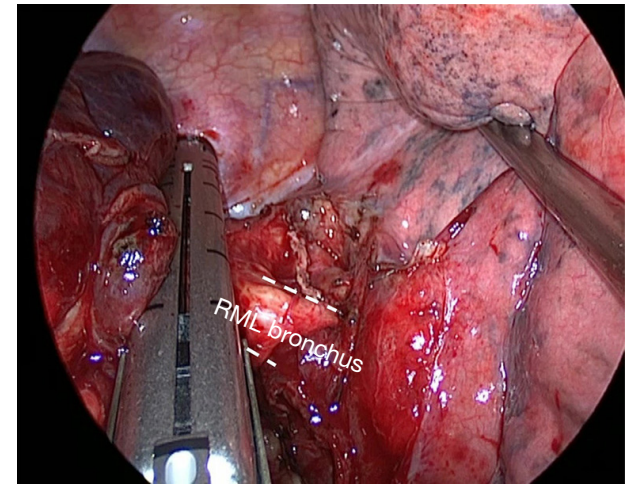

Figure 6 Checking middle-lobe ventilation while clamping the right-lower bronchus with the stapler. RML, right middle lobe.

the bronchial time, he can even divide together both the LLB and the posterior fissure with thick staplers. Figure 4 resumes the main steps for right-lower lobectomy.

Most of the endostaplers placed during lower lobectomies can be placed straight or with mild angulation; less experienced surgeons sometimes feel that they need

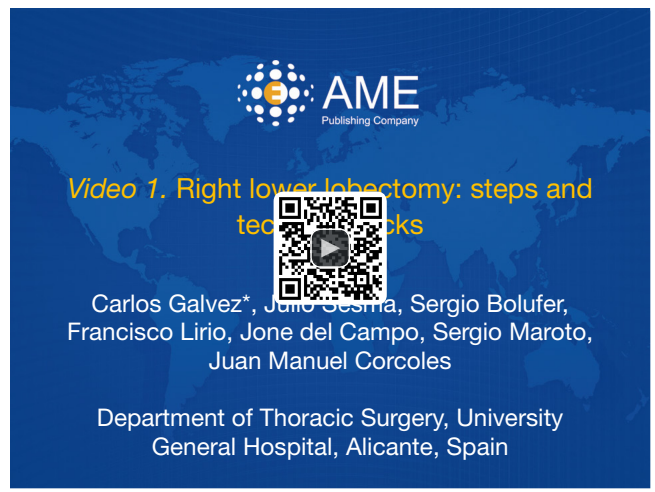

Figure 7 Right lower lobectomy: steps and technical tricks (16). Available online: http://www.asvide.com/watch/32945

more angulation for some steps but essentially it just requires more exhaustive dissection and ability to go ahead with the procedure in the order that makes easier normal positioning of the staplers. Figure 7 summarizes main steps of right-lower lobectomy with almostcomplete fissure. 


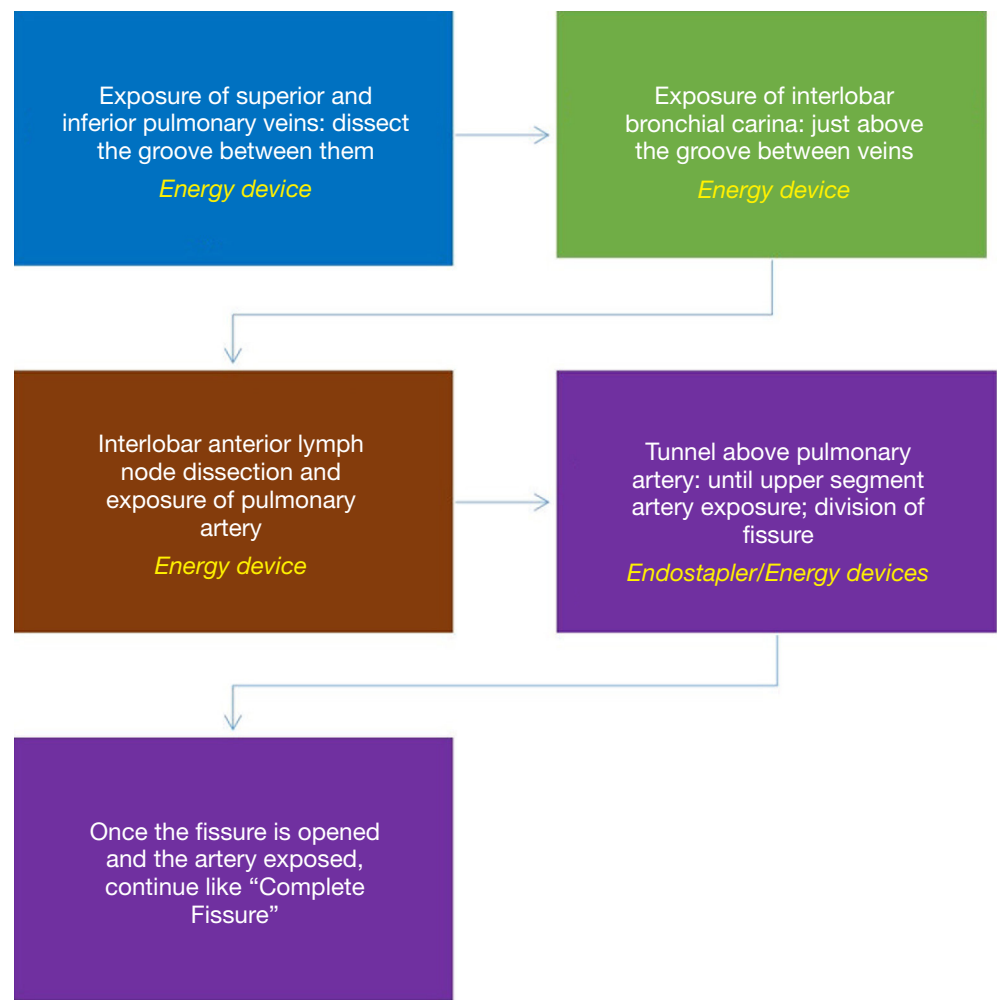

Figure 8 Right-lower lobectomy (incomplete fissure).

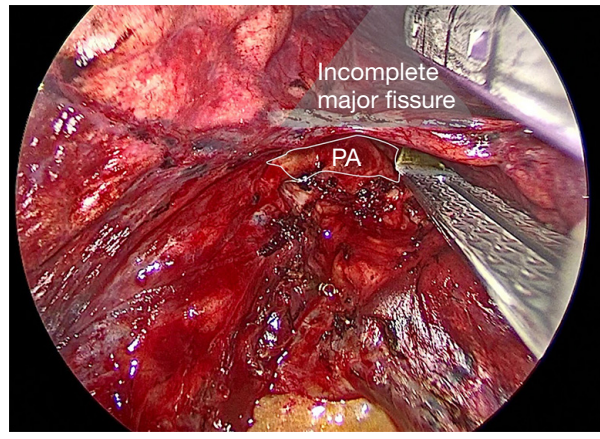

Figure 9 "Tunnel” technique during right-lower lobectomy with incomplete fissure. PA, pulmonary artery.

\section{Incomplete fissure (Craig Walker III-IV) (Figure 8)}

Some lungs present with absence or minimal major fissure, so dissection of the artery results more difficult. We here describe how to convert an incomplete fissure into a complete one. Pulling the lung posteriorly, we dissect the groove between superior and inferior pulmonary vein: there surgeon can find immediately above the interlobar bronchial division, which is just below the pulmonary artery in the fissure. Once you identify the artery, dissect above it getting deeper as a "tunnel" and, as long as you expose the artery you can divide the fissure above the artery with consecutive endostaplers or energy devices (Figure 9). When the surgeon has already divided the fissure above the artery the procedure continues exactly as in the complete fissure description.

Some surgeons prefer the fissureless technique for incomplete fissures, but we do not recommend this technique unless the unique option. Fissureless technique means dividing the fissure at the end when the other bronchovascular structures have already been divided from caudal to cranial. First divide the pulmonary ligament and inferior pulmonary vein. Then, dissect the bronchus, but this is the most dangerous step, specially in less experienced surgeons: while dissecting the bronchus from down to up, you must keep the tip of the dissector strictly in contact with the bronchus because if not, you can tear the pulmonary artery that lays behind.

We find safer and more advisable converting the incomplete fissure into a complete one, whose trick consists on dissecting from down to up the groove between the veins, then the groove between the bronchi and finally identifying the artery as described and using the "tunnel" 
technique to divide the fissure.

\section{Specimen removal}

We recommended that the specimen is taken out with a specimen retrieval bag, which is an easy step. We usually

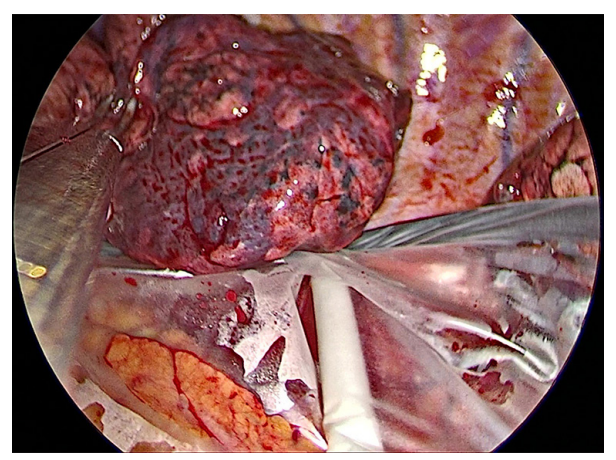

Figure 10 Specimen retrieval. grasp the specimen and pull it upwards close to the ribs, and introduce the bag below the specimen (Figure 10). Then we deliver the bag from the introductor system and as it opens, we let the specimen fall inside and then the assistant can push into the bag the specimen with the suction. Just close the bag and remove it through the utility incision.

\section{Right lymphadenectomy (Figure 11)}

\section{Right lower paratracheal area (4R)}

For right lower paratracheal lymphadenectomy, the assistant stands at the left of the surgeon and combines both the camera and the suction to pull upwards the mediastinal pleura once the surgeon has already open it at this area. We usually open the pleura just above the entrance of azygous vein into superior vena cava, and continue dividing superiorly parallel to the superior vena cava. Then we grasp together the lymph nodes and the fatty tissue with a node grasper, and we resect the tissue with energy devices

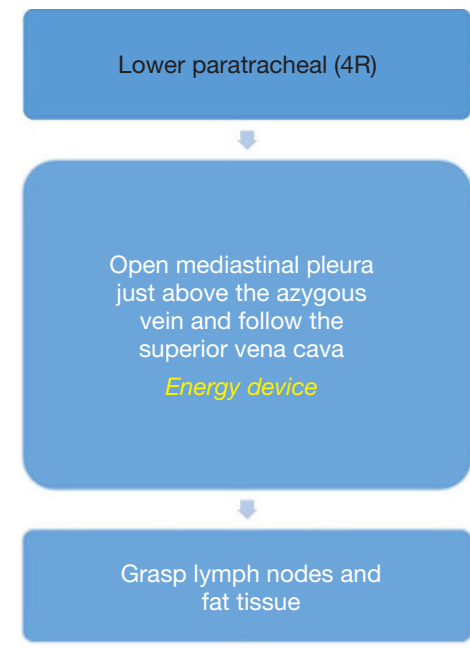

果

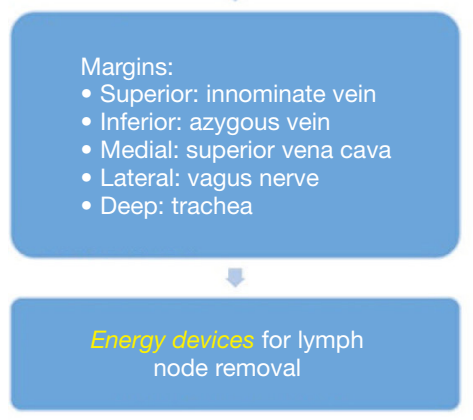

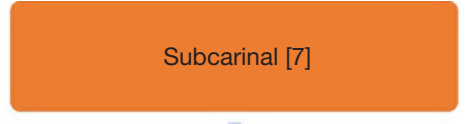

$\sqrt{5}$

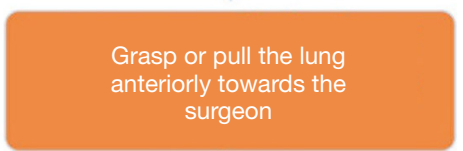

$\nabla$

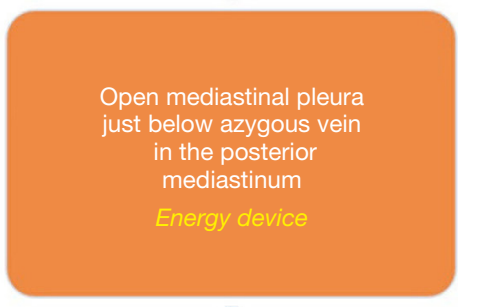

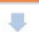

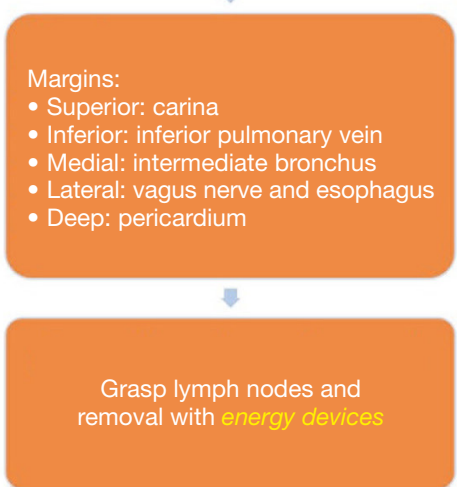

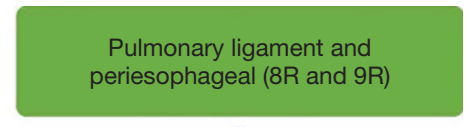

8

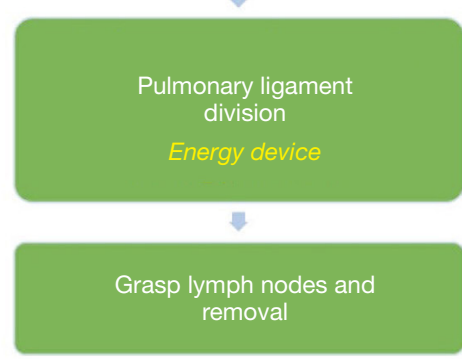

Figure 11 Right lymphadenectomy. 

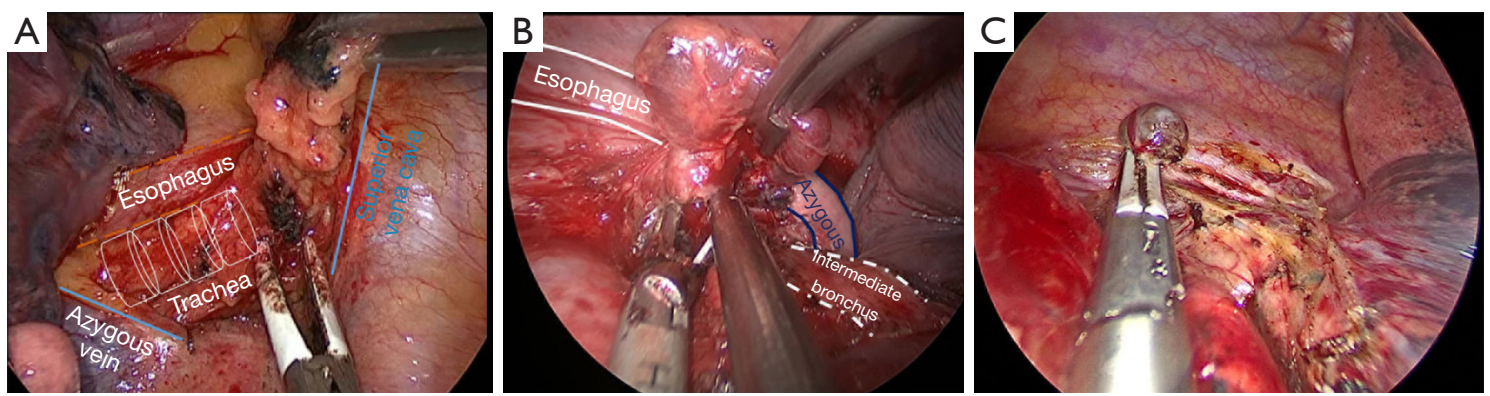

Figure 12 Right side lymphadenectomy. (A) Right lower paratracheal area (4R); (B) subcarinal dissection [7]; (C) pulmonary ligament and periesophageal dissection (8R and 9R).

following these margins: inferiorly, azygous vein; medial margin, superior vena cava; lateral margin, vagus nerve; superiorly, innominate vein; deep margin, the trachea (Figure 12A). If dissection gets deeper by the anterior aspect of the trachea, pretracheal lymph nodes (level 3) can be included. Chylothorax or bleeding from small vessels are the most common complications while performing exhaustive $4 \mathrm{R}$ lymphadenectomies.

\section{Subcarinal area [7]}

For the subcarinal area, first rotate the operating table towards the surgeon anteriorly so the lung falls slightly anteriorly, and then the surgeon pulls the lung towards himself with a sponge stick with his left hand, and with the right hand continues dividing the posterior mediastinal pleura from inferior pulmonary vein until carina with energy devices. We prefer that the assistant stands on the right of the surgeon, cranial and close to the flexed arms, but positioning on both sides of the surgeon can be useful depending on the patient's anatomy. Assistant can use the suction for pulling upwards the mediastinal pleura. The surgeon grasps the lymph nodes in the area and resects the tissue keeping in mind the posterior limit of this area (esophagus), and deeply the pericardium (Figure 12B). Remind the systemic vascularization from the aorta to the bronchial circulation and lymph nodes, which can bleed notably. Subcarinal space is one of the most troublesome areas for lymphadenectomy and if exhaustive can reach the contralateral hemithorax, although it's easier to be performed in the right side than in the left one.

\section{Periesophageal and pulmonary ligament (8R and 9R)}

These stations can be safely dissected while dividing the pulmonary ligament, and are more easily dissected with the assistant standing on the right of the surgeon, cranial and close to the flexed arms (Figure 12C).

\section{Left lower lobectomy}

Here it should also be highlighted the difference between those with complete or almost complete fissure, and those with incomplete fissure.

\section{Complete or almost complete fissure (I-II Craig Walker classification) (Figure 13)}

Left lower lobectomy with complete fissure is probably the easiest lobectomy. It can be usually performed in four consecutive steps: arterial division, fissure completion, inferior vein division and bronchial division. There's logically no need for special care with middle lobe bronchus and arteries. Specific care should be taken while exposing the arterial trunk for basilar segments, to identify the lingular artery, that although not frequent, can raise from the basilar arterial trunk in the lower lobe to reach the lingula. The procedure can be performed following the same recommendations of right-lower lobectomy. Most of the times the artery can be safely divided with a sole vascular endostapler for both the upper segment artery and the basilar trunk. Figure 14 summarizes main steps of leftlower lobectomy with almost-complete fissure.

\section{Incomplete fissure (II-IV Craig Walker classification) (Figure 15)}

In contrast with complete fissure left-lower lobectomy, cases with incomplete fissure can be challenging. As previously mentioned, we consider safer and useful trying to switch the incomplete fissure into a complete one rather than performing the lobectomy in a fissureless approach. 


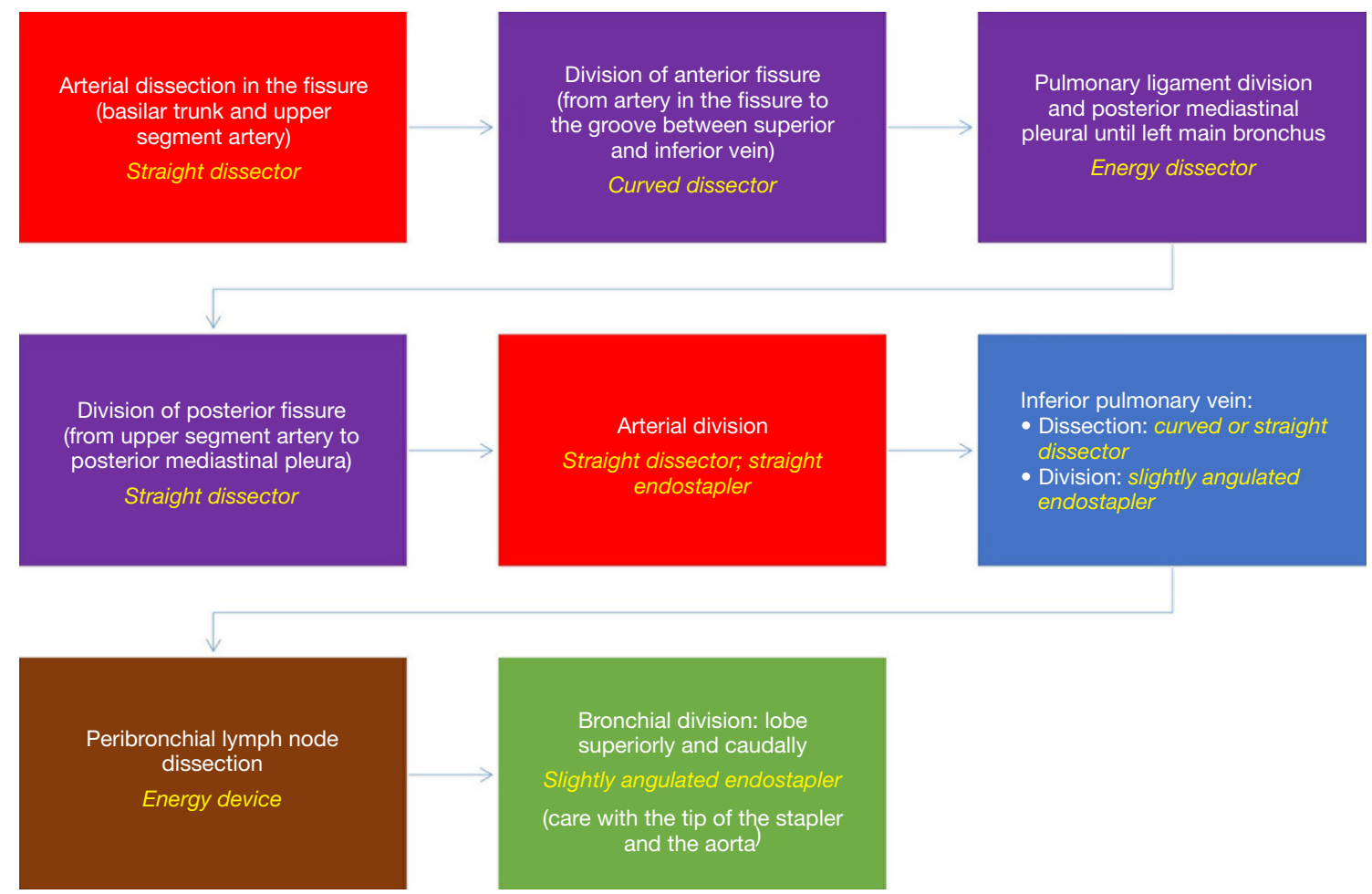

Figure 13 Left-lower lobectomy (complete fissure).

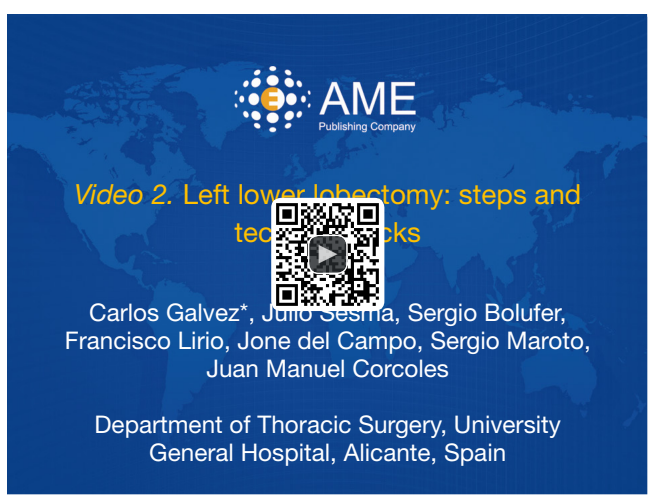

Figure 14 Left lower lobectomy: steps and technical tricks (17). Available online: http://www.asvide.com/watch/32946

If you find very difficult to expose the artery in the fissure, grasp the anterior fissure and pull the lung backwards to expose the hilum: dissect the mediastinal fat to expose both the superior and inferior pulmonary vein (check always that there are two veins and not a unique vein). In the groove between both veins, try to dissect the lung parenchyma distally upwards to expose the interlobar bronchial carina (between left upper bronchus and left lower bronchus). Immediately above this carina, you will discover interlobar lymph nodes (11L) that after careful dissection expose the pulmonary artery. Then dissect above the artery with the "tunnel" technique and divide progressively the fissure with endostaplers to avoid air leaks. Once you expose the upper segment artery (A6), you can dissect the left lower artery with a straight dissector, and divide it with a vascular stapler (care with the aorta). Usually the endostapler can be placed without angulation if the incision is placed in the right intercostal space, preferably the $6^{\text {th }}$ space.

For pulmonary ligament and inferior pulmonary vein division we prefer the assistant placed in the cranial position close to the flexed arms. We divide the ligament and then continue dissecting towards the subcarinal station, to expose the posterior aspect of the inferior vein and identify the vein for the upper segment.

Then, pull the lobe upwards towards the chest wall and dissect anteriorly the vein easily. We find also useful the straight dissector for this step, but in some cases with big tumors where you can't pull upwards the lobe as much as desired, a long-curved dissector can be preferable. After that, the vein can be divided with a vascular stapler: position the stapler with mild angulation of the tip superiorly to 


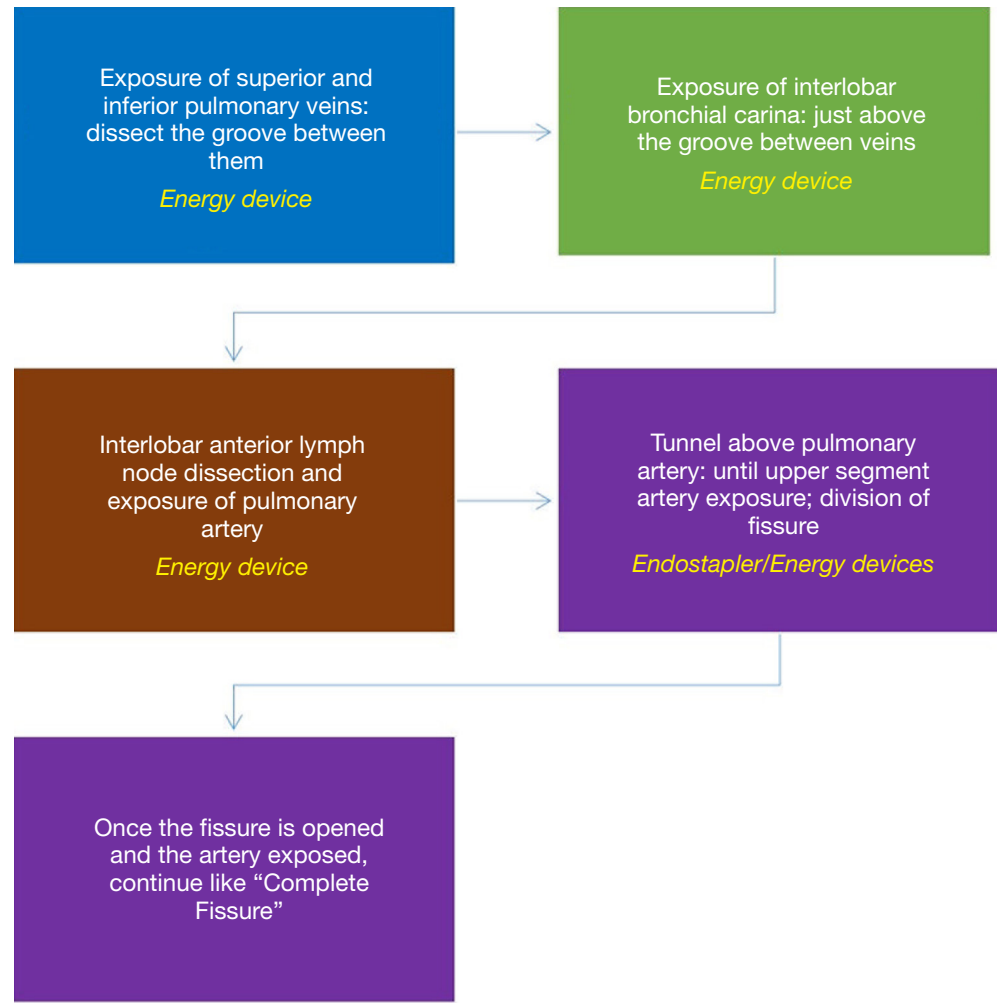

Figure 15 Left-lower lobectomy (incomplete fissure).
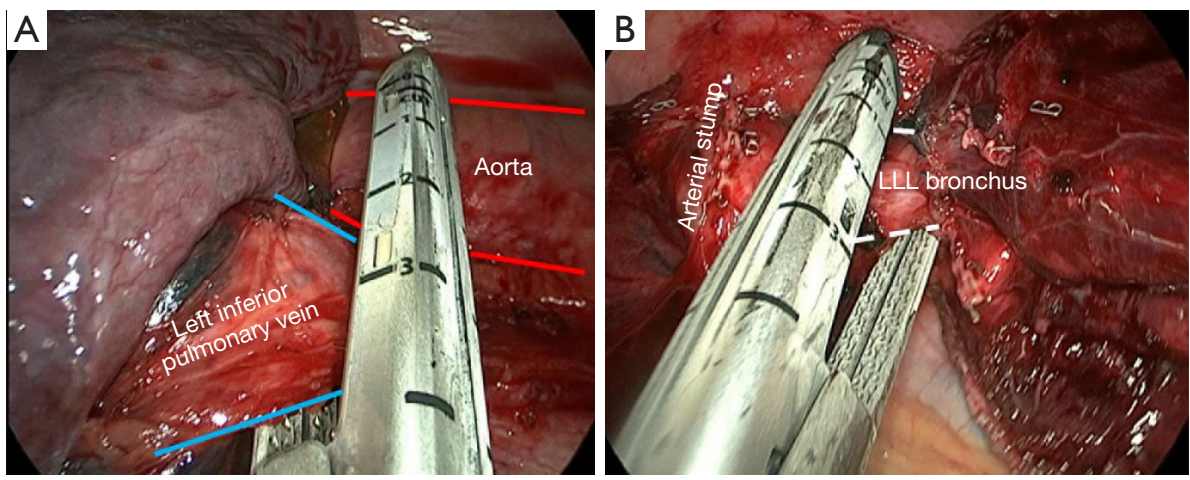

Figure 16 Left-lower lobectomy. (A) Positioning of the vascular stapler for IPV division; (B) lower bronchus division with special care to the aorta behind. IPV, inferior pulmonary vein; LLL, left lower lobe.

avoid the descending aorta (Figure 16A). It's not infrequent to find the upper segment vein (V6) raising separately and very close to the posterior mediastinal pleura, so be careful not to damage the upper segment vein with the tip.

After vascular division, by pulling the lung caudally, surgeon can dissect the lymph nodes surrounding the leftlower bronchus below the vascular arterial and venous stumps, and divide it using a thick endostapler. Keep in mind that from uniportal incision, the tip of the stapler points to the descending aorta, so mild angulation of the stapler can be useful for this purpose (Figure 16B). Figure 11 summarizes main steps in left-lower lobectomy.

Left-lower lobectomy can be performed in a fissureless technique, more easily than the right-lower lobectomy. After division of the pulmonary ligament, the vein can be exposed, dissected and divided in the same way than in the technique 


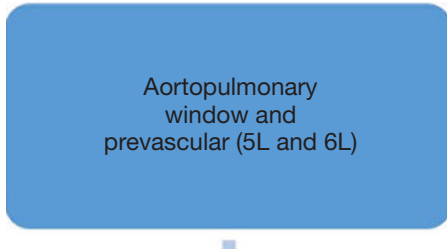

吝

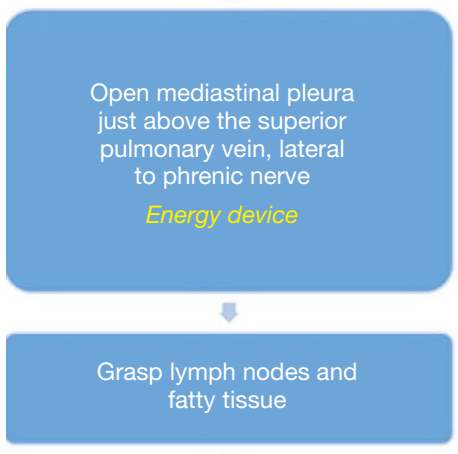

常

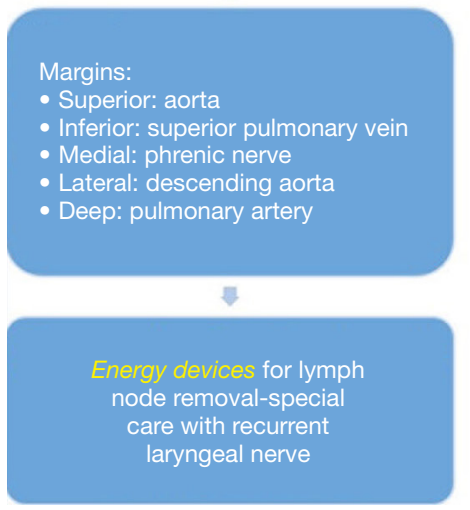

Figure 17 Left lymphadenectomy.

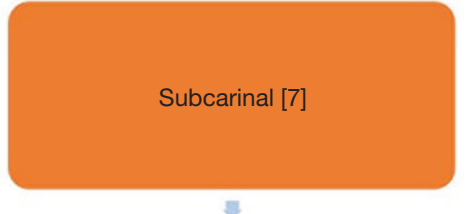

$\sqrt{8}$
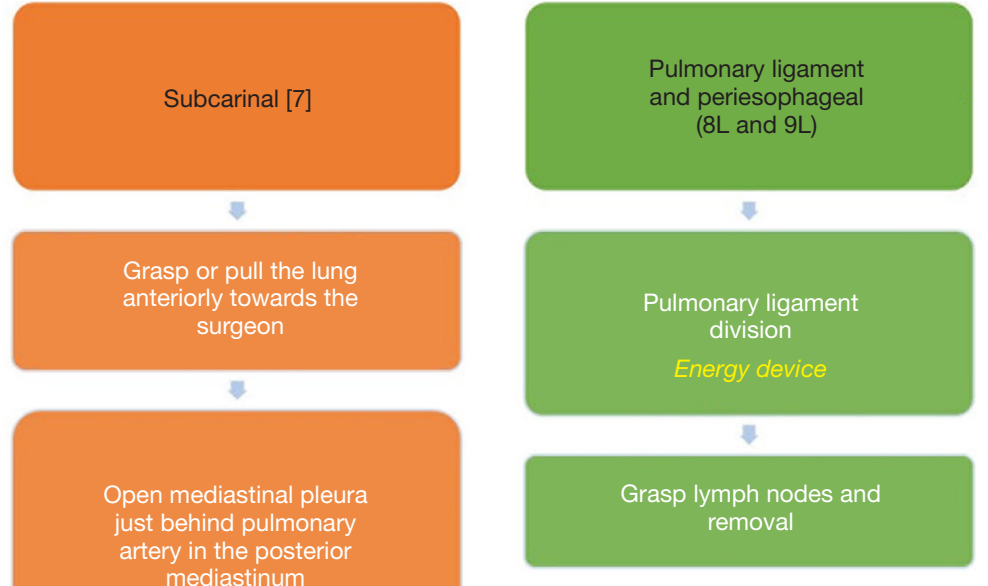

periesophag division

Energy device

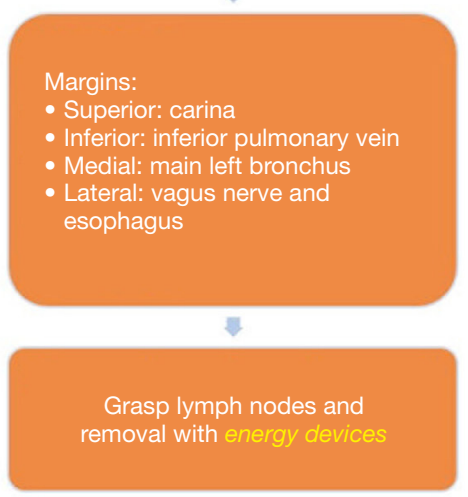

mediastinum

Energy device

분
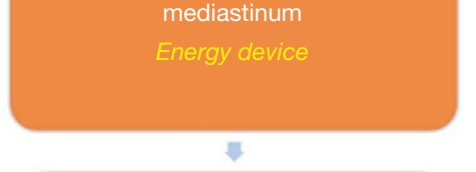

\section{政}
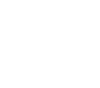

above described. Then the bronchus can be dissected from below: for this purpose, I find useful the Gonzalez-Rivas dissector (almost right-angle), keeping the tip during the whole step close to the bronchus. Once the bronchus has been divided, the artery lies on the left, in a view from below. Pulling the lung cranially you can dissect the artery with a right-angle dissector and then divide it with a vascular endostapler. Identify before the division, the lingular artery which indicates where to place the vascular endostapler for the left-lower artery. Finally, when all the structures have been divided, place the lung in its normal position and just follow the theoretical fissure with thick endostaplers for completing the lobectomy in a fissureless approach.

\section{Specimen removal}

It's recommended that the specimen is taken out with a specimen retrieval bag, which is an easy step. We usually grasp the specimen and pull it upwards close to the ribs, and introduce the bag below the specimen. Then we deliver the bag from the introductor system and as it opens, we let the specimen fall inside and then the assistant can push into the bag the specimen with the suction. Just close the bag and remove it through the utility incision.

\section{Left lymphadenectomy (Figure 17)}

Prevascular and aortopulmonary window (5L, 6L)

This area can be dissected in a similar way than right lower paratracheal area, with the assistant standing on the right of the surgeon. Dissection should be limited anteriorly by phrenic nerve, cranially by aortic arch and the laryngeal recurrent nerve, and deeper by the pulmonary artery (Figure 18A). Caution should focus on avoiding laryngeal 

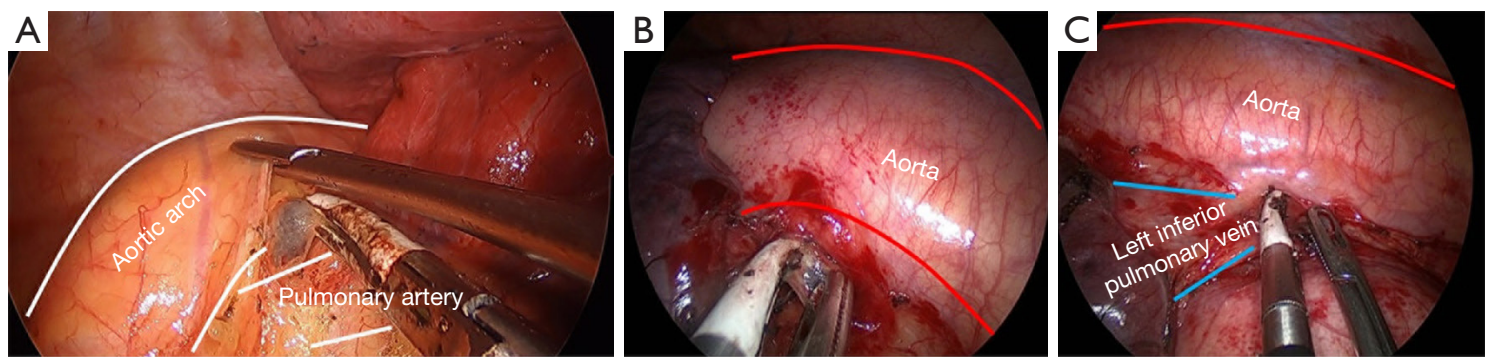

Figure 18 Left-side lymphadenectomy. (A) Aortopulmonary window (5L); (B) subcarinal dissection [7]; (C) pulmonary ligament and periesophageal dissection (8L and 9L).

recurrent nerve damage while dissecting lymph nodes below the aortic arch, and always keep in mind the balance benefit/risk between radical dissection and severity of potential laryngeal nerve damage. If dissection continues towards posterior hilum, just behind the left pulmonary artery surgeon may reach the deep left lower paratracheal area $(4 \mathrm{~L})$.

\section{Subcarinal area [7]}

This is probably the most difficult area during uniportal VATS lymphadenectomy. After posterior mediastinal pleura division from the pulmonary ligament until the pulmonary artery, pull the lung anteriorly with a sponge stick and rotate the operating table towards the surgeon. Grasp the lymph nodes and fatty tissue altogether and resect it with energy devices (Figure 18B). Keep in mind the vagus nerve behind, and deeply the esophagus.

\section{Periesophageal and pulmonary ligament (8L and 9L)}

These stations can be safely dissected while dividing the pulmonary ligament (Figure 18C), and are more easily dissected with the assistant standing on the left of the surgeon, cranial and close to the flexed arms.

\section{Conclusions}

Lower lobectomies, specially with complete fissures, are the most suitable procedures for starting with uniportal VATS lobectomies. They can be safely and easily performed through this approach.

Some specific tips and tricks should be taken into account for ensuring surgeon's comfort and patient's safety. The position of both the surgeon and the assistant should be interchanged during these procedures to avoid crashing between them, and shoulder injuries in the assistant.

Lymphadenectomies can be safely performed although moderate experience is required for ensuring oncological principles.

Uniportal VATS claims urgently for detailed description of specific steps by experienced groups, and standardization to homogenize the technique, define the limits, and set a didactic method for teaching non-experienced surgeons and residents.

\section{Acknowledgments}

To my beloved wife for listening patiently my passionate explanations of my uniportal progression through these 5 years since I started with anatomical major resections; for her lovely support and trust in me.

To my wonderful surgical team, including surgeons, residents, anesthesiologists and nurses, because our team work has positioned us on the top of the wave.

To everyone who goes ahead with passion, trying always to improve and succeed, but knows that success is only temporary and it's the attitude what defines you.

\section{Footnote}

Conflicts of Interest: The authors have no conflicts of interest to declare.

Informed Consent: Written informed consent was obtained from the patient for publication of this manuscript and any accompanying images.

\section{References}

1. Gonzalez D, Paradela M, Garcia J, et al. Single-port videoassisted thoracoscopic lobectomy. Interact Cardiovasc Thorac Surg 2011;12:514-5.

2. Gonzalez-Rivas D, de la Torre M, Fernandez R, 
et al. Single-port video-assisted thoracoscopic left upper lobectomy. Interact Cardiovasc Thorac Surg 2011;13:539-41.

3. Chen YY, Lin TH, Chang CC, et al. Staged bronchial closure in uniportal video-assisted thoracoscopic anatomical resection for lung cancer with calcified lymph nodes. J Vis Surg 2017;3:149.

4. Ismail M, Swierzy M, Nachira D, et al. Uniportal videoassisted thoracic surgery for major lung resections: Pitfalls, tips and tricks. J Thorac Dis 2017;9:885-97.

5. Sihoe A. Uniportal video-assisted thoracoscopic lobectomy versus other video-assisted thoracoscopic lobectomy techniques: A randomized study. Ann Thorac Surg 2018;50:411-5.

6. Wei S, Chen N, Liu C, et al. Does single-port videoassisted thoracic lobectomy have favorable perioperative results for non-small cell lung cancer compared with multi-port approach? A systematic review and metaanalysis. Video-assist Thorac Surg 2017;2:33.

7. Abouarab AA, Rahouma M, Kamel M, et al. Single Versus Multi-Incisional Video-Assisted Thoracic Surgery: A Systematic Review and Meta-analysis. J Laparoendosc Adv Surg Tech A 2018;28:174-85.

8. Yang Z, Zhai C. Uniportal video-assisted thoracoscopic surgery following neoadjuvant chemotherapy for locallyadvanced lung cancer. J Cardiothorac Surg 2018;13:33.

9. Hernandez-Arenas LA, Purmessur RD, Gonzalez-Rivas D. Uniportal video-assisted thoracoscopic segmentectomy. J Thorac Dis 2018;10:S1205-14.

Cite this article as: Galvez C, Sesma J, Bolufer S, Lirio F, del Campo J, Maroto S, Corcoles JM. The techniques of uniportal video-assisted thoracoscopic surgery: lower lobectomies and lymphadenectomy. J Thorac Dis 2019;11(Suppl 16):S2095-S2107. doi: $10.21037 /$ jtd.2019.02.67
10. Anile M, Diso D, Mantovani S, et al. Uniportal video assisted thoracoscopic lobectomy: Going directly from open surgery to a single port approach. J Thorac Dis 2014;6:S641-3.

11. Aragón J, Méndez IP. From open surgery to uniportal VATS: Asturias experience. J Thorac Dis 2014;6:S644-9.

12. Bertolaccini L, Viti A, Terzi A, et al. Geometric and ergonomic characteristics of the uniportal video-assisted thoracoscopic surgery (VATS) approach. Ann Cardiothorac Surg 2016;5:118-22.

13. Martin-Ucar AE, Aragon J, Bolufer Nadal S, et al. The influence of prior multiport experience on the learning curve for single-port thoracoscopic lobectomy: a multicentre comparative study $\dagger$. Eur J Cardiothorac Surg 2017;51:1183-7.

14. Craig SR, Walker WS. A proposed anatomical classification of the pulmonary fissures. J R Coll Surg Edinb 1997;42:233-4.

15. Nagashima T, Shimizu K, Ohtaki Y, et al. Analysis of variation in bronchovascular pattern of the right middle and lower lobes of the lung using three-dimensional CT angiography and bronchography. Gen Thorac Cardiovasc Surg 2017;65:343-9.

16. Galvez C, Sesma J, Bolufer S, et al. Right lower lobectomy: steps and technical tricks. Asvide 2019;6:260. Available online: http://www.asvide.com/watch/32945

17. Galvez C, Sesma J, Bolufer S, et al. Left lower lobectomy: steps and technical tricks. Asvide 2019;6:261. Available online: http://www.asvide.com/watch/32946 